\title{
Approximation Algorithms for Multi-Criteria Traveling Salesman Problems*
}

\author{
Bodo Manthey ${ }^{\dagger, 1}$ \\ L. Shankar Ram ${ }^{2}$ \\ ${ }^{1}$ Yale University \\ Department of Computer Science \\ P. O. Box 208285, New Haven, CT 06520-8285, USA \\ manthey@cs.yale.edu \\ ${ }^{2}$ ETH Zürich \\ Institut für Theoretische Informatik \\ 8092 Zürich, Switzerland \\ shankar.lakshminarayanan@ag.ch
}

\begin{abstract}
We analyze approximation algorithms for several variants of the traveling salesman problem with multiple objective functions. First, we consider the symmetric TSP (STSP) with $\gamma$-triangle inequality. For this problem, we present a deterministic polynomial-time algorithm that achieves an approximation ratio of $\min \{1+$ $\left.\gamma, \frac{2 \gamma^{2}}{2 \gamma^{2}-2 \gamma+1}\right\}+\varepsilon$ and a randomized approximation algorithm that achieves a ratio of $\frac{2 \gamma^{3}+2 \gamma^{2}}{3 \gamma^{2}-2 \gamma+1}+\varepsilon$. In particular, we obtain a $2+\varepsilon$ approximation for multi-criteria metric STSP.

Then we show that multi-criteria cycle cover problems admit fully polynomial-time randomized approximation schemes. Based on these schemes, we present randomized approximation algorithms for STSP with $\gamma$-triangle inequality (ratio $\frac{1+\gamma}{1+3 \gamma-4 \gamma^{2}}+\varepsilon$ ), asymmetric TSP (ATSP) with $\gamma$-triangle inequality (ratio $\frac{1}{2}+\frac{\gamma^{3}}{1-3 \gamma^{2}}+\varepsilon$ ), STSP with weights one and two (ratio 4/3) and ATSP with weights one and two (ratio 3/2).
\end{abstract}

\section{Introduction}

In many practical optimization problems, there is not only one single objective function to measure the quality of a solution, but there are several such functions. Consider for instance buying a car: We (probably) want to buy a cheap car that is fast and has a good gas mileage. How do we decide which car is the best one for us? Of course, with respect to any single criterion, making the decision is easy. But with multiple criteria involved, there is no natural notion of a best choice. The aim of multi-criteria optimization (also called multi-objective optimization or Pareto optimization) is to cope with this problem. To transfer the concept of a best choice to multi-criteria optimization, the notion of Pareto curves was introduced (cf. Section 1.1 and Ehrgott [12]). A Pareto curve is a set of solutions that can be considered optimal.

However, for most optimization problems, Pareto curves cannot be computed efficiently. Thus, we have to be content with approximations to them.

\footnotetext{
${ }^{*}$ A preliminary version of this work has been presented at the 4th Workshop on Approximation and Online Algorithms (WAOA 2006) [20].

${ }^{\dagger}$ Supported by the Postdoc-Program of the German Academic Exchange Service (DAAD). On leave from Saarland University. Work done in part at the Institute for Theoretical Computer Science of the University of Lübeck supported by DFG research grant RE 672/3 and at the Department of Computer Science at Saarland University.
} 
The traveling salesman problem (TSP) is one of the best-known combinatorial optimization problems $[16,18]$. An instance of the TSP is a complete graph with edge weights, and the aim is to find a Hamiltonian cycle (also called a tour) of minimum weight. Since the TSP is NP-hard [14], we cannot hope to always find an optimal tour efficiently. For practical purposes, however, it is often sufficient to obtain a tour that is close to optimal. In such cases, we require approximation algorithms, i. e., polynomial-time algorithms that compute such near-optimal tours.

While the approximability of several variants of the single-criterion TSP has been studied extensively in the past decades, not much is known about the approximability of multi-criteria TSP. The classical TSP is about a traveling salesman who has to visit a certain number of cities and return back home in a shortest tour. "Real" saleswomen and salesmen do not face such a simple situation. Instead, while arranging their tours, they have to bear in mind several objectives that are to be optimized. For instance, the distance traveled and the travel time should be minimized while the journey should be as cheap as possible. This gives rise to multi-criteria TSP, for which we design approximation algorithms in this paper.

\section{$1.1 \quad$ Preliminaries}

Graphs and Optimization Problems. Let $G=(V, E)$ be a graph (directed or undirected) with edge weights $w: E \rightarrow \mathbb{N}$. We define the weight of a subgraph $G^{\prime}=\left(V^{\prime}, E^{\prime}\right)$ of $G$ or a subset $E^{\prime}$ of the edges of $G$ as the sum of the weights of its edges: $w\left(G^{\prime}\right)=$ $w\left(E^{\prime}\right)=\sum_{e \in E^{\prime}} w(e)$. For $k \in \mathbb{N}$, we define $[k]=\{1,2, \ldots, k\}$.

TSP in general is the following optimization problem: Given a graph with edge weights, find a Hamiltonian cycle, i. e., a cycle that visits every vertex of the graph exactly once, of minimum weight. In case of undirected graphs, we speak of the symmetric TSP (STSP), while in case of directed graphs, we refer to the problem as the asymmetric TSP (ATSP).

An instance of $\boldsymbol{\Delta}$-STSP is an undirected complete graph $G=(V, E)$ with edge weights $w: E \rightarrow \mathbb{N}$ that fulfill triangle inequality, i. e., $w(\{u, v\}) \leq w(\{u, x\})+w(\{x, v\})$ for all distinct vertices $u, v, x \in V$.

For $\gamma \in\left[\frac{1}{2}, 1\right], \boldsymbol{\Delta}(\gamma)$-STSP is the restriction of $\Delta$-STSP to instances that satisfy $\gamma$ strengthened triangle inequality, i. e., $w(\{u, v\}) \leq \gamma \cdot(w(\{u, x\})+w(\{x, v\}))$ for all distinct vertices $u, v, x$.

$\operatorname{STSP}(1,2)$ is the special case of $\Delta$-STSP where only one and two are allowed as edge weights, i. e., $w: E \rightarrow\{1,2\}$.

$\Delta$-ATSP $\boldsymbol{\Delta}(\gamma)$-ATSP, and $\operatorname{ATSP}(1,2)$ are defined like their undirected counterparts $\Delta$-STSP, $\Delta(\gamma)$-STSP, and $\operatorname{STSP}(1,2)$, respectively, except that the graphs are directed.

Note that for $\gamma=1, \Delta(\gamma)$-STSP and $\Delta(\gamma)$-ATSP become $\Delta$-STSP and $\Delta$-ATSP, respectively. As $\gamma$ gets smaller, the edge weights become more and more structured. For $\gamma=1 / 2$, all edge weights are equal. The $\gamma$-strengthened triangle inequality can also be considered as a data-dependent bound [7]: Given an instance of metric TSP, we compute the minimum $\gamma$ such that the instance fulfills $\gamma$-strengthened triangle inequality. If $\gamma<1$, then we obtain a better performance guarantee for our approximate solution than with triangle inequality alone.

A cycle cover of a graph $G=(V, E)$ is a subgraph $(V, C)$ that consists solely of cycles such that every vertex $v \in V$ is part of exactly one cycle. In most cases, we refer to a cycle cover as the set $C$ of its edges. Hamiltonian cycles are cycle covers that consist of only a single cycle. 
The problem of computing cycle covers of minimum weight in undirected graphs is called SCC. The directed version of the problem is called ACC.

Multi-Criteria Optimization. A $k$-criteria optimization problem consists of a set $I$ of instances, a set $\operatorname{sol}(x)$ of feasible solutions for every instance $x \in I, k$ objective functions $w_{1}, \ldots, w_{k}$, each mapping pairs of $x \in I$ and $y \in \operatorname{sol}(x)$ to $\mathbb{N}$, and $k$ types indicating whether $w_{i}$ should be minimized or maximized. We refer to Ehrgott and Gandibleux [12, 13] for surveys on multi-criteria optimization problems. Throughout this paper, we restrict ourselves to problems where all objective functions should be minimized. Furthermore, we assume that the number $k$ of criteria is fixed. The running-times of our algorithms are exponential in $k$. But since $k$ is typically a small number, this does not cause any harm.

The optimization problems defined in Section 1.1 are generalized to their multi-criteria counterparts in the obvious way: We have $k$ objective functions $w_{1}, \ldots, w_{k}$, each induced by edge weight functions (to which we also refer as $w_{1}, \ldots, w_{k}$ ) as described. If we have additional restrictions on the edge weights, like the triangle inequality, every edge weight function is assumed to fulfill them.

In general, the different objective functions are in conflict with each other, i. e., it is impossible to minimize all of them simultaneously. Therefore, the notion of Pareto curves has been introduced. For the following definitions, let $\Pi$ be a $k$-criteria optimization problem as defined above.

A set $\mathcal{P}(x) \subseteq \operatorname{sol}(x)$ is called a Pareto curve of $x$ if for all solutions $z \in \operatorname{sol}(x)$, there exists a solution $y \in \mathcal{P}(x)$ with $w_{i}(x, y) \leq w_{i}(x, z)$ for all $i \in[k]$.

A Pareto curve contains all solutions that might be considered optimal. If there are two solutions $y$ and $z$ with $w_{i}(x, y)=w_{i}(x, z)$ for all $i \in[k]$, then it suffices to put one of them into $\mathcal{P}(x)$. For completeness, let us mention that Pareto curves are not unique in general: In our definition, it is not forbidden to include dominated solutions in $\mathcal{P}(x)$ (a solution $y$ is dominated if there exists a $z$ with $w_{i}(x, z) \leq w_{i}(x, y)$ for all $i \in[k]$ and $w_{i}(x, z)<w_{i}(x, y)$ for some $i \in[k]$, i. e., $z$ is strictly better than $\left.y\right)$.

For the majority of multi-criteria problems, computing Pareto curves is hard for two reasons: First, many two-criteria problems allow for a reduction from the knapsack problem. Second, Pareto curves are often of exponential size. Therefore, we have to be content with approximate Pareto curves. Let $\beta \geq 1$, and let $x \in I$ and $\mathcal{P}^{\text {apx }}(x) \subseteq \operatorname{sol}(x)$. The set $\mathcal{P}^{\text {apx }}(x)$ is called a $\boldsymbol{\beta}$-approximate Pareto curve for $x$ if, for every $z \in \operatorname{sol}(x)$, there exists a $y \in \mathcal{P}^{\text {apx }}(x)$ with $w_{i}(x, y) \leq \beta \cdot w_{i}(x, z)$ for all $i \in[k]$.

A 1-approximate Pareto curve is a Pareto curve. For completeness, let us mention that if $\Pi$ is a maximization problem (or an objective $w_{i}$ for some $i \in[k]$ should be maximized), then the condition is $w_{i}(x, z) \leq \beta \cdot w_{i}(x, y)$.

While Pareto curves itself are often of exponential size, it is known that $(1+\varepsilon)$ approximate Pareto curves of size polynomial in the input size and $1 / \varepsilon$ exist [23]. (The technical restriction is that the objective functions are restricted to assume values of at most $2^{p(|x|)}$ for $x \in I$ and some polynomial $p$.)

The above definition leads immediately to the notion of an approximation algorithm for multi-criteria optimization problems: Let $\beta \geq 1$. A $\boldsymbol{\beta}$-approximation algorithm for $\Pi$ is an algorithm that, for every input $x \in I$, computes a $\beta$-approximate Pareto curve for $x$ in time polynomial in the size $|x|$ of $x$.

A randomized $\boldsymbol{\beta}$-approximation algorithm for $\Pi$ is a polynomial-time algorithm that, for every input $x \in I$, computes a set $\mathcal{P}^{\text {apx }}(x) \subseteq \operatorname{sol}(x)$ such that $\mathcal{P}^{\text {apx }}(x)$ is a $\beta$-approximate Pareto curve for $x$ with a probability of at least $1 / 2$.

By executing a randomized approximation algorithm $\ell$ times, we obtain a $\beta$-appro- 


\begin{tabular}{|l|c|l|}
\hline Variant & Ratio & Reference \\
\hline$\Delta$-STSP & $3 / 2$ & Christofides [10] \\
\hline$\Delta(\gamma)$-STSP & $\min \left\{\frac{3 \gamma^{2}}{3 \gamma^{2}-2 \gamma+1}, \frac{2-\gamma}{3-3 \gamma}\right\}$ & Böckenhauer et al. [8] \\
\hline STSP $(1,2)$ & $8 / 7$ & Berman, Karpinski [5] \\
\hline$\Delta$-ATSP & $0.842 \cdot \log n$ & Kaplan et al. [17] \\
\hline$\Delta(\gamma)$-ATSP & $\min \left\{\frac{1+\gamma}{2-\gamma-\gamma^{3}}, \frac{\gamma}{1-\gamma}\right\}$ & Bläser et al. [7]; Chandran and Ram [9] \\
\hline $\operatorname{ATSP}(1,2)$ & $5 / 4$ & Bläser [6] \\
\hline
\end{tabular}

Table 1: Approximability of single-criterion TSP.

ximate Pareto curve with a probability of at least $1-2^{-\ell}$, i. e., the failure probability tends exponentially to zero: We take the union of all sets of solutions computed in the $\ell$ iterations and throw away all solutions that are dominated by solutions in the union.

Given the notion of (randomized) approximation algorithms, we can define approximation schemes. A fully polynomial-time approximation scheme (FPTAS) for $\Pi$ is an algorithm that, on input $x \in I$ and $\varepsilon>0$, computes a $(1+\varepsilon)$-approximate Pareto curve in time polynomial in the size of $x$ and $1 / \varepsilon$.

A fully polynomial-time randomized approximation scheme (FPRAS) for $\Pi$ is a randomized approximation algorithm that, on input $x \in I$ and $\varepsilon>0$, computes a $(1+\varepsilon)$-approximate Pareto curve in time polynomial in the size of $x$ and $1 / \varepsilon$.

Finally, we define the notion of a randomized exact algorithm: A randomized exact algorithm for $\Pi$ is an algorithm that, on input $x$, computes a Pareto curve of $x$ in time polynomial in the size of $x$ with a probability of at least $1 / 2$.

An optimization problem $\Pi$ is said to be polynomially bounded if there exists a polynomial $p$ such that the following holds for every objective function $w_{i}$ of $\Pi$ : For every instance $x$ and every feasible solution $y$ for $x, w_{i}(x, y) \leq p(|x|)$ for all $i \in[k]$. Analogously to the fact that a polynomially bounded single-criterion problem that admits an FPTAS can be solved exactly in polynomial time (cf. Ausiello et al. [3, Theorem 3.15]), randomized exact algorithms exist for polynomially bounded multi-criteria optimization problems that admit an FPRAS.

\subsection{Previous Results}

The approximability of single-criterion TSP has been studied intensively in the past. Table 1 shows the currently best approximation ratios of the variants for which the multicriteria counterparts are considered in this paper.

While single-criterion optimization problems and their approximation properties have been the subject of a considerable amount of research (cf. Ausiello et al. [3] for a survey), not much is known about the approximability of multi-criteria optimization problems.

Papadimitriou and Yannakakis [23], by applying results of Barahona and Pulleyblank [4], Mulmuley et al. [21], and themselves [22], showed that there exist FPTASs for multi-criteria minimum-weight spanning trees and the multi-criteria shortest path problem and an FPRAS (more precisely, a fully polynomial RNC scheme) for the multi-criteria minimum weight matching problem. The results were established by showing that a multicriteria problem admits an FPTAS if the exact version of the single-criterion problem can be solved in pseudo-polynomial time. Let $\Pi$ be a single-criterion optimization problem with instance set $I$ and objective function $w$. The exact version of $\boldsymbol{\Pi}$ is the following decision problem: Given an instance $x \in I$ and a number $W \in \mathbb{N}$, does there exist a solution $y \in \operatorname{sol}(x)$ with $w(x, y)=W$ ? 
The exact versions of many single-criterion optimization problems are NP-complete since knapsack can be reduced to them easily. But this does not rule out the possibility of pseudo-polynomial-time algorithms for them.

Multi-criteria TSP has been investigated by Ehrgott [11] and Angel et al. [1,2]. Ehrgott [11] considered a generalization of Christofides' algorithm for $\Delta$-STSP. Instead of considering Pareto curves, he measured the quality of a solution $y$ for an instance $x$ as a norm of the vector $\left(w_{1}(x, y), \ldots, w_{k}(x, y)\right)$. Thus, he encoded the different objective functions into a single one, which reduces the problem to a single-criterion problem. The approximation ratio achieved is between $3 / 2$ and 2, depending on the norm used to combine the different criteria. However, by encoding all objective functions into a single one, we lose the special properties of multi-criteria optimization problems.

Angel et al. [1] considered two-criteria $\operatorname{STSP}(1,2)$. They presented a 3/2-approximation algorithm for this problem by using a local search heuristic. Finally, Angel et al. [2] generalized these results to $k$-criteria $\operatorname{STSP}(1,2)$ by presenting a $2-\frac{2}{k+1}$-approximation for $k \geq 3$. Although for every fixed $k$, the approximation ratio is below 2 , it converges to 2 as $k$ increases. Thus, the ratio tends to the trivial ratio of 2 , which can be achieved by selecting any Hamiltonian cycle. These two are the only papers about the approximability of Pareto curves of multi-criteria TSP we are aware of.

\subsection{Our Results}

All our results hold for an arbitrary but fixed number of objective functions.

We present a deterministic polynomial-time algorithm that computes $(2+\varepsilon)$-approximate Pareto curves for $\Delta$-STSP (Section 2.1). This is the first efficient algorithm for computing approximate Pareto curves for this problem. In fact, we show the following more general result: If the edge weights satisfy $\gamma$-strengthened triangle inequality for $\gamma \in\left[\frac{1}{2}, 1\right]$, then the algorithm computes a $\left(\min \left\{1+\gamma, \frac{2 \gamma^{2}}{2 \gamma^{2}-2 \gamma+1}\right\}+\varepsilon\right)$-approximate Pareto curve for arbitrarily small $\varepsilon>0$ in polynomial time.

We generalize Christofides' algorithm [10] (cf. Vazirani [26, Sect. 3.2]) to obtain a randomized approximation algorithm for multi-criteria $\Delta(\gamma)$-STSP (Section 2.2). For $\gamma \in\left[\frac{1}{2}, 1\right]$, our algorithm achieves an approximation performance of $\frac{2 \gamma^{3}+2 \gamma^{2}}{3 \gamma^{2}-2 \gamma+1}+\varepsilon$. For $\gamma=1$, this yields a ratio of $2+\varepsilon$.

We consider cycle covers in Section 3. Cycle covers play an important role in the design of approximation algorithms for the TSP. We prove that there exists an FPRAS for computing approximate Pareto curves of multi-criteria cycle covers. Subsequently, we extend this result and show that the multi-criteria variant of the problem of finding graph factors of minimum weight admits an FPRAS, too.

Finally, we analyze a randomized cycle-cover-based algorithm for multi-criteria TSP (Section 4): We start by computing an approximate Pareto curve of cycle covers. Then, for every cycle cover in the set computed, we remove one edge of every cycle and join the paths thus obtained to a Hamiltonian cycle. We analyze the approximation ratio of this algorithm for $\Delta(\gamma)$-STSP (Section 4.2, approximation ratio $\frac{1+\gamma}{1+3 \gamma-4 \gamma^{2}}+\varepsilon$ for $\gamma<1$ ), $\Delta(\gamma)$-ATSP (Section 4.3, ratio $\frac{1}{2}+\frac{\gamma^{3}}{1-3 \gamma^{2}}+\varepsilon$ for $\left.\gamma<1 / \sqrt{3}\right), \operatorname{STSP}(1,2)$, and $\operatorname{ATSP}(1,2)$ (Section 4.4, ratios $4 / 3$ and $3 / 2$, respectively).

As far as we know, our algorithms are the first approximation algorithms for Pareto curves for $\Delta$-STSP, $\Delta(\gamma)$-STSP, $\Delta(\gamma)$-ATSP, and $\operatorname{ATSP}(1,2)$. Furthermore, we achieve a better approximation ratio for $\operatorname{STSP}(1,2)$ than the approximation algorithms by Angel et al. $[1,2]$ for all $k$. 


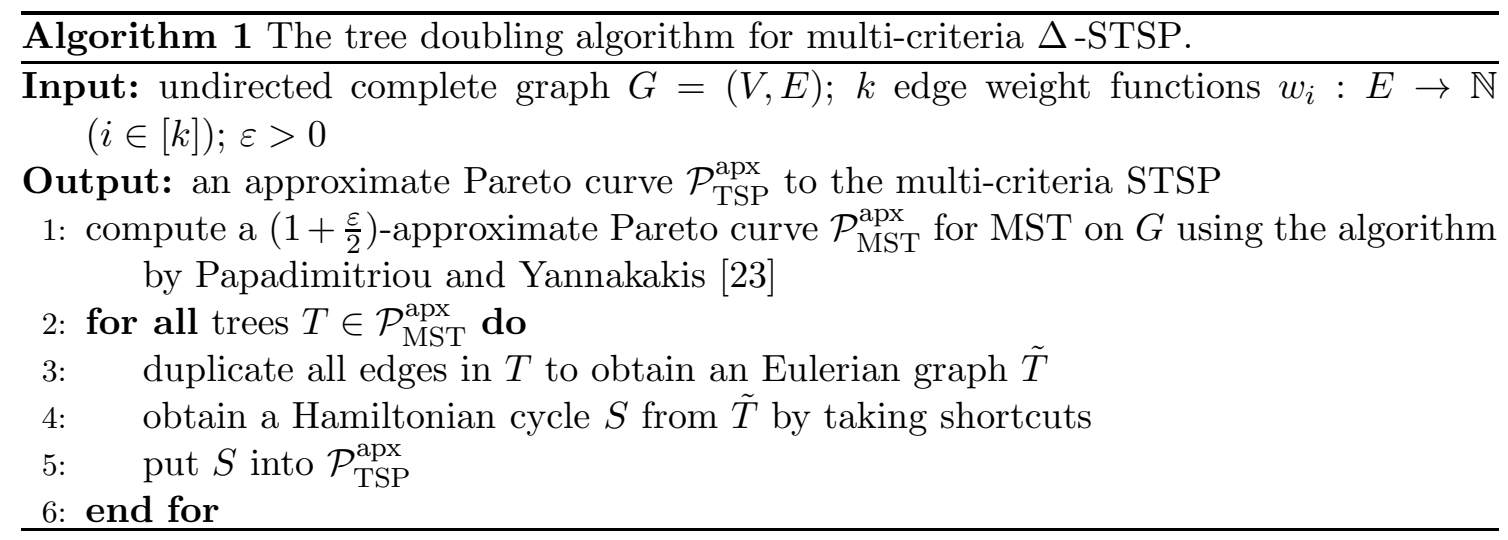

\section{Metric TSP}

In this section, we present two algorithms for $\Delta$-STSP and $\Delta(\gamma)$-STSP. Another approximation algorithm that can be used for approximating $\Delta(\gamma)$-STSP, which is based on computing cycle covers, will be presented in Section 4.

The analyses of the algorithms in this section exploit the following result due to Böckenhauer et al. [8].

Lemma 2.1 (Böckenhauer et al. [8]). Let $G=(V, E)$ be an undirected complete graph with an edge weight function $w$ satisfying $\gamma$-strengthened triangle inequality for some $\gamma \in\left[\frac{1}{2}, 1\right)$.

Let $w_{\max }=\max _{e \in E}(w(e))$ and $w_{\min }=\min _{e \in E}(w(e))$ be the weights of a heaviest and lightest edge, respectively. Then $\frac{w_{\max }}{w_{\min }} \leq \frac{2 \gamma^{2}}{1-\gamma}$.

Let $e$ and $e^{\prime}$ be two edges with a common endpoint. Then $\frac{w(e)}{w\left(e^{\prime}\right)} \leq \frac{\gamma}{1-\gamma}$.

Furthermore, we observe the following: Omitting two edges by taking a shortcut reduces the weight by at least $2 \cdot(1-\gamma) \cdot w_{\min }$ : The reason is that the two edges $(u, v)$ and $(v, x)$ are replaced by $(u, x)$ and $w(u, x) \leq \gamma \cdot(w(u, v)+w(v, x))$. Thus, the weight is reduced by at least $w(u, v)+w(v, x)-w(u, x) \geq(1-\gamma) \cdot(w(u, v)+w(v, x)) \geq 2 \cdot(1-\gamma) \cdot w_{\min }$.

\subsection{The Generalized Tree Doubling Algorithm}

Consider the following approximation algorithm for single-criterion $\Delta$-STSP, which was first analyzed by Rosenkrantz et al. [24] (cf. Vazirani [26, Sect. 3.2]): First, we compute a minimum spanning tree. Then we duplicate each edge. The result is an Eulerian graph. We obtain a Hamiltonian cycle from this graph by walking along an Eulerian cycle. If we come back to a vertex that we have already visited, we omit it and take a short-cut to the next vertex in the Eulerian cycle. In this way, we obtain an approximation ratio of 2 for single-criterion $\Delta$-STSP. Algorithm 1 is an adaptation of this algorithm to multi-criteria STSP. In the following, we estimate the approximation performance of this algorithm.

Theorem 2.2. For all $\gamma \in\left[\frac{1}{2}, 1\right]$, Algorithm 11 computes a $\left(\min \left\{1+\gamma, \frac{2 \gamma^{2}}{2 \gamma^{2}-2 \gamma+1}\right\}+\varepsilon\right)$ approximate Pareto curve for multi-criteria $\Delta(\gamma)$-STSP in time polynomial in the input size and $1 / \varepsilon$.

Proof. We present two analyses showing approximation ratios of $1+\gamma+\varepsilon$ and $\frac{2 \gamma^{2}}{2 \gamma^{2}-2 \gamma+1}+\varepsilon$, respectively. The first analysis holds for $\gamma \in\left[\frac{1}{2}, 1\right]$ while the second one only holds for $\gamma \in\left[\frac{1}{2}, 1\right)$. However, $1+\gamma=\frac{2 \gamma^{2}}{2 \gamma^{2}-2 \gamma+1}$ for $\gamma=1$. 
The key observation of the first analysis is the following: Let $T \in \mathcal{P}_{\mathrm{MST}}^{\mathrm{apx}}$, and let $e$ be any edge in $T$. Then $e$ appears twice in $\tilde{T}$, but $e$ cannot appear twice in $S$ since $S$ is a Hamiltonian cycle. (We assume that $G$ contains at least three vertices.) Thus, at least one copy of $e$ is omitted. This is the moment at which the strengthened triangle inequality comes into play. Let $e_{1}, e_{2}, \ldots, e_{\ell}$ with $e_{j}=\left\{v_{j-1}, v_{j}\right\}$ be a path along the Eulerian cycle in $\tilde{T}$ such that this path is replaced by the edge $\left\{v_{0}, v_{\ell}\right\}$ by taking a shortcut. Then we have

$$
w_{i}\left(\left\{v_{0}, v_{\ell}\right\}\right) \leq \gamma \cdot\left(w_{i}\left(e_{1}\right)+w_{i}\left(e_{2}\right)+\ldots+w_{i}\left(e_{\ell}\right)\right)
$$

by iteratively applying $\gamma$-strengthened triangle inequality. (We exploit the fact that $\gamma^{c} \leq \gamma$ for all $c \geq 1$.) Overall, every edge that we omit contributes at most a fraction of $\gamma$ of its weight. Since we omit at least one copy of every edge $e$, the two copies of $e$ contribute at most $(1+\gamma) \cdot w_{i}(e)$ to $S$. Thus,

$$
w_{i}(S) \leq(1+\gamma) \cdot w_{i}(T)
$$

for all $i \in[k]$.

To estimate the overall approximation performance, let $S^{\prime}$ be an arbitrary Hamiltonian cycle. By omitting one edge, we obtain a tree $T^{\prime}$. Since $\mathcal{P}_{\text {MST }}^{\text {apx }}$ is a $(1+\varepsilon / 2)$-approximate Pareto curve for multi-criteria minimum-weight spanning trees on $G$, there exists a tree $T \in \mathcal{P}_{\text {MST }}^{\text {apx }}$ with

$$
w_{i}(T) \leq\left(1+\frac{\varepsilon}{2}\right) \cdot w_{i}\left(T^{\prime}\right) \leq\left(1+\frac{\varepsilon}{2}\right) \cdot w_{i}\left(S^{\prime}\right)
$$

for all $i \in[k]$. Let $S$ be the Hamiltonian cycle obtained from $T$, then

$$
w_{i}(S) \leq(1+\gamma) \cdot w_{i}(T) \leq(1+\gamma+\varepsilon) \cdot w_{i}\left(S^{\prime}\right)
$$

for all $i \in[k]$.

For the second analysis, let again $S^{\prime}$ be an arbitrary Hamiltonian cycle. This analysis only holds for $\gamma<1$ since $w_{\max } / w_{\min }$ can be unbounded for $\gamma=1$. All arguments hold simultaneously for all criteria $i \in[k]$. Without loss of generality, we assume that $\min _{e \in E} w_{i}(e)=1$ for all $i \in[k]$, i. e., $w_{\min }=1$. By removing one edge of $S^{\prime}$, we obtain a tree with a weight of at most $w_{i}\left(S^{\prime}\right)-w_{\min }=w_{i}\left(S^{\prime}\right)-1$. Thus, there exists a tree $T \in \mathcal{P}_{\text {MST }}^{\text {apx }}$ from which we obtain a Eulerian graph $\tilde{T}$ with

$$
w_{i}(\tilde{T}) \leq 2 \cdot\left(1+\frac{\varepsilon}{2}\right) \cdot\left(w_{i}\left(S^{\prime}\right)-1\right)=(2+\varepsilon) \cdot\left(w_{i}\left(S^{\prime}\right)-1\right) .
$$

Let $n=|V|$ be the number of vertices of the whole graph. Then $\tilde{T}$ contains $2 n-2$ edges. Thus, in order to obtain a Hamiltonian cycle, we have to remove $n-2$ edges by taking shortcuts. Every shortcut decreases the weight by at least $2 \cdot(1-\gamma)$. Hence,

$$
\begin{aligned}
w_{i}(S) & \leq w_{i}(\tilde{T})-(n-2) \cdot 2 \cdot(1-\gamma) \\
& \leq(2+\varepsilon) \cdot w_{i}\left(S^{\prime}\right)-(2+\varepsilon)-(n-2) \cdot 2 \cdot(1-\gamma) \\
& \leq(2+\varepsilon) \cdot w_{i}\left(S^{\prime}\right)-2 n \cdot(1-\gamma)-(4 \gamma-2) \\
& \leq(2+\varepsilon) \cdot w_{i}\left(S^{\prime}\right)-2 n \cdot(1-\gamma)
\end{aligned}
$$

since $2 \gamma \geq 1$. We have $w_{i}\left(S^{\prime}\right)=\frac{2 n \gamma^{2}}{(1-\gamma) \cdot \alpha}$ for some appropriately chosen $\alpha \geq 1$, which implies

$$
\frac{w_{i}(S)}{w_{i}\left(S^{\prime}\right)} \leq 2+\varepsilon-\frac{2 n \cdot(1-\gamma)^{2} \cdot \alpha}{2 \gamma^{2} n}=2+\varepsilon-\frac{(1-\gamma)^{2} \cdot \alpha}{\gamma^{2}} \text {. }
$$




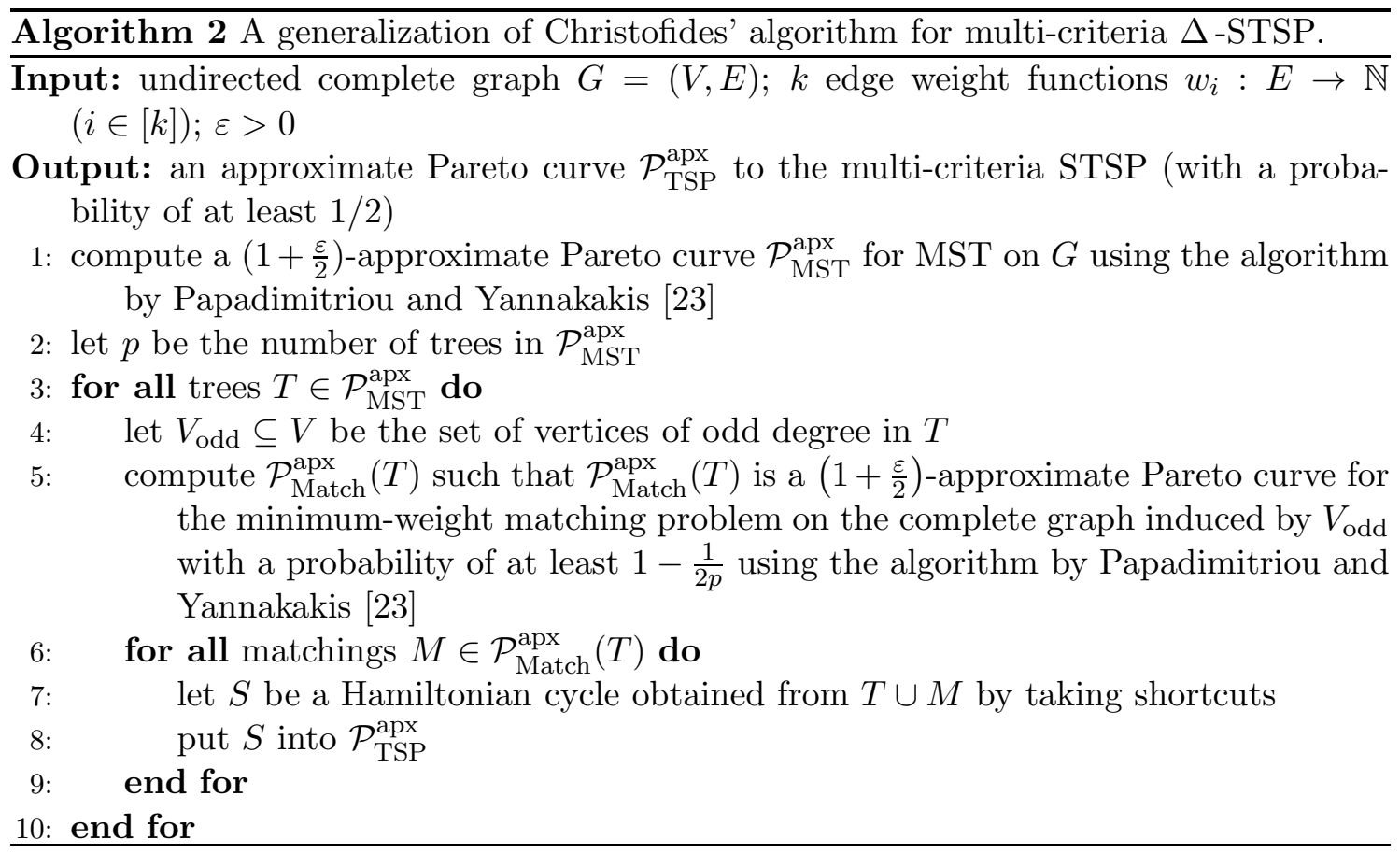

Since $w_{i}(S) \leq n \frac{2 \gamma^{2}}{1-\gamma}$, we also have $\frac{w_{i}(S)}{w_{i}\left(S^{\prime}\right)} \leq \alpha$. Thus, the approximation ratio achieved is

$$
\begin{aligned}
& \max _{\alpha \geq 1}\left(\min \left(\alpha, 2+\varepsilon-\frac{(1-\gamma)^{2} \cdot \alpha}{\gamma^{2}}\right)\right) \\
\leq & \max _{\alpha \geq 1}\left(\min \left(\alpha, 2-\frac{(1-\gamma)^{2} \cdot \alpha}{\gamma^{2}}\right)\right)+\varepsilon \\
= & \frac{2 \gamma^{2}}{2 \gamma^{2}-2 \gamma+1}+\varepsilon,
\end{aligned}
$$

which completes the proof of the theorem.

For small values of $\gamma$, the bound of $\frac{2 \gamma^{2}}{2 \gamma^{2}-2 \gamma+1}+\varepsilon$ is the stronger one, while $1+\gamma+\varepsilon$ yields a better bound in case of $\gamma>1 / \sqrt{2}$.

Corollary 2.3. Algorithm 1 computes $(2+\varepsilon)$-approximate Pareto curves for multi-criteria $\Delta$-STSP in time polynomial in the input size and $1 / \varepsilon$.

\subsection{A Generalization of Christofides' Algorithm}

In this section, we generalize Christofides' algorithm to multi-criteria $\Delta$-STSP, which is the best approximation algorithm for single-criterion $\Delta$-STSP known so far. This algorithm computes approximate Pareto curves of matchings. In case of single-criterion $\Delta$-STSP, we can always find a matching with a weight of at most half of the weight of the optimal Hamiltonian cycle. This is in contrast to multi-criteria $\Delta$-STSP, where the weights of the matchings can be arbitrarily close to the weight of the optimal Hamiltonian cycle. The reason is that we cannot choose the lighter of two different matchings since multiple objective functions are involved; the term "'lighter"' is not well defined. Therefore, we only get an approximation ratio of roughly two in this case. But for $\Delta(\gamma)$-STSP, we can show a better upper bound. 
Theorem 2.4. For $\gamma \in\left[\frac{1}{2}, 1\right]$, Algorithm 2 is a randomized $\left(\frac{2 \gamma^{3}+2 \gamma^{2}}{3 \gamma^{2}-2 \gamma+1}+\varepsilon\right)$-approximation algorithm for multi-criteria $\Delta(\gamma)$-STSP. Its running time is polynomial in the input size and $1 / \varepsilon$.

Proof. The proof consists of two parts. First, we estimate the approximation performance, given that all Pareto curves computed are $(1+\varepsilon / 2)$-approximate Pareto curves. Second, we estimate the success probability, i. e., the probability that such a Pareto curve is computed.

We assume that all Pareto curves that have to be computed during the execution of the algorithm were computed successfully, i. e., with an appropriate approximation ratio. Let $S^{\prime}$ be an arbitrary Hamiltonian cycle of $G$. Without loss of generality, we assume again that $w_{\min }=1$. All the arguments in the following hold for all $i$.

There exists a tree $T \in \mathcal{P}_{\text {MST }}^{\text {apx }}$ with

$$
w_{i}(T) \leq\left(1+\frac{\varepsilon}{2}\right) \cdot\left(w_{i}\left(S^{\prime}\right)-1\right)
$$

Let $V_{\text {odd }}$ be the set of vertices of odd degree in $T$, and let $n_{\text {odd }}$ be its cardinality. Note that $n_{\text {odd }}$ is even, thus perfect matchings exist on the complete graph induced by $V_{\text {odd }}$. Let $n=|V|$ be the number of vertices of the whole graph. Let $S_{\text {odd }}$ be the Hamiltonian cycle obtained from $S^{\prime}$ by taking shortcuts. We get two matchings $M_{1}$ and $M_{2}$ on $V_{\text {odd }}$ from $S_{\text {odd }}$ by putting the edges of $S_{\text {odd }}$ alternately into $M_{1}$ and $M_{2}$. By $\gamma$-triangle inequality, we have

$$
w_{i}\left(M_{1}\right)+w_{i}\left(M_{2}\right)=w_{i}\left(S_{\text {odd }}\right) \leq w_{i}\left(S^{\prime}\right)-\left(n-n_{\text {odd }}\right) \cdot 2 \cdot(1-\gamma)
$$

since every shortcut reduces the weight by at least $(1-\gamma) \cdot 2 w_{\min }$. Now, $w_{i}\left(M_{1}\right) \cdot \frac{1-\gamma}{\gamma} \leq$ $w_{i}\left(M_{2}\right)$ according to Lemma 2.1. (Note that we do not make any assumptions whether $M_{1}$ or $M_{2}$ is the lighter matching.) There exists a matching $M \in \mathcal{P}_{\text {Match }}^{\text {apx }}(T)$ with

$$
w_{i}(M) \leq\left(1+\frac{\varepsilon}{2}\right) \cdot w_{i}\left(M_{1}\right) .
$$

Hence,

$$
\begin{aligned}
\frac{w_{i}(M)}{\gamma}=w_{i}(M) \cdot\left(1+\frac{1-\gamma}{\gamma}\right) & \leq\left(1+\frac{\varepsilon}{2}\right) \cdot\left(w_{i}\left(M_{1}\right)+w_{i}\left(M_{2}\right)\right) \\
& \leq\left(1+\frac{\varepsilon}{2}\right) \cdot\left(w_{i}\left(S^{\prime}\right)-2 \cdot\left(n-n_{\text {odd }}\right) \cdot(1-\gamma)\right) .
\end{aligned}
$$

Let $D$ be Eulerian graph obtained by taking the union of the tree $T$ and the matching $M$. By the arguments above, we can bound its weight as follows:

$$
\begin{aligned}
w_{i}(D) & =w_{i}(T)+w_{i}(M) \\
& \leq\left(1+\frac{\varepsilon}{2}\right) \cdot\left(w_{i}\left(S^{\prime}\right)-1+\gamma \cdot\left(w_{i}\left(S^{\prime}\right)-2 \cdot\left(n-n_{\text {odd }}\right) \cdot(1-\gamma)\right)\right) \\
& =\left(1+\frac{\varepsilon}{2}\right) \cdot\left((1+\gamma) \cdot w_{i}\left(S^{\prime}\right)-\left(1+2 \gamma \cdot\left(n-n_{\text {odd }}\right) \cdot(1-\gamma)\right)\right) .
\end{aligned}
$$

The Eulerian graph $D$ consists of $n-1+n_{\text {odd }} / 2$ edges, the tour $S$ constructed from $D$ consists only of $n$ edges. Thus, $n_{\text {odd }} / 2-1$ edges are removed and, by $\gamma$-triangle inequality, we have

$$
w_{i}(S) \leq w_{i}(D)-2 \cdot\left(n_{\text {odd }} / 2-1\right) \cdot(1-\gamma)=w_{i}(D)-(1-\gamma) \cdot\left(n_{\text {odd }}-2\right) .
$$


By combining these inequalities, we obtain

$$
\begin{aligned}
w_{i}(S) \leq & \left(1+\frac{\varepsilon}{2}\right) \cdot\left((1+\gamma) \cdot w_{i}\left(S^{\prime}\right)\right. \\
& \left.\quad-\left(1+2 \gamma \cdot\left(n-n_{\text {odd }}\right) \cdot(1-\gamma)+(1-\gamma) \cdot\left(n_{\text {odd }}-2\right)\right)\right) \\
= & \left(1+\frac{\varepsilon}{2}\right) \cdot\left((1+\gamma) \cdot w_{i}\left(S^{\prime}\right)\right. \\
& \left.\quad-(\underbrace{1-2+2 \gamma}_{\geq 0}+(1-\gamma) \cdot(\underbrace{2 \gamma}_{\geq 1} \cdot\left(n-n_{\text {odd }}\right)+n_{\text {odd }}))\right) \\
\leq & \left(1+\frac{\varepsilon}{2}\right) \cdot\left((1+\gamma) \cdot w_{i}\left(S^{\prime}\right)-n \cdot(1-\gamma)\right) .
\end{aligned}
$$

Now we have $w_{\max } \leq \frac{2 \gamma^{2}}{1-\gamma}$ since $w_{\min }=1$. Thus, $w_{i}\left(S^{\prime}\right) \leq \frac{2 \gamma^{2}}{1-\gamma} \cdot n$. We choose $\alpha \geq 1$ such that $w_{i}\left(S^{\prime}\right)=\frac{2 n \gamma^{2}}{(1-\gamma) \cdot \alpha}$, which implies

$$
\begin{aligned}
\frac{w_{i}(S)}{w_{i}\left(S^{\prime}\right)} & \leq\left(1+\frac{\varepsilon}{2}\right) \cdot\left((1+\gamma)-\frac{n \cdot(1-\gamma)}{w_{i}\left(S^{\prime}\right)}\right) \leq\left(1+\frac{\varepsilon}{2}\right) \cdot\left((1+\gamma)-\frac{\alpha n \cdot(1-\gamma)}{\frac{2 \gamma^{2}}{1-\gamma} \cdot n}\right) \\
& =\left(1+\frac{\varepsilon}{2}\right) \cdot\left((1+\gamma)-\frac{\alpha(1-\gamma)^{2}}{2 \gamma^{2}}\right) \leq(1+\gamma)-\frac{\alpha(1-\gamma)^{2}}{2 \gamma^{2}}+\varepsilon .
\end{aligned}
$$

The last inequality holds since $(1+\gamma)-\frac{\alpha(1-\gamma)^{2}}{2 \gamma^{2}} \leq 1+\gamma \leq 2$. is

Since $w_{i}(S) \leq n \frac{2 \gamma^{2}}{1-\gamma}$, we also have $\frac{w_{i}(S)}{w_{i}\left(S^{\prime}\right)} \leq \alpha$. Thus, the approximation ratio achieved

$$
\begin{aligned}
& \max _{\alpha \geq 1}\left(\min \left(\alpha,(1+\gamma)-\frac{\alpha(1-\gamma)^{2}}{2 \gamma^{2}}+\varepsilon\right)\right) \\
\leq & \frac{2 \gamma^{3}+2 \gamma^{2}}{3 \gamma^{2}-2 \gamma+1}+\varepsilon \cdot \frac{2 \gamma^{2}}{3 \gamma^{2}-2 \gamma+1} \leq \frac{2 \gamma^{3}+2 \gamma^{2}}{3 \gamma^{2}-2 \gamma+1}+\varepsilon .
\end{aligned}
$$

We obtain the first inequality by observing that $(1+\gamma)-\frac{\alpha(1-\gamma)^{2}}{2 \gamma^{2}}+\varepsilon$ is monotonically decreasing in $\alpha$ : The maximum of the minimum is therefore assumed for $\alpha=(1+\gamma)-$ $\frac{\alpha(1-\gamma)^{2}}{2 \gamma^{2}}+\varepsilon$. The second inequality follows from the fact that $\frac{2 \gamma^{2}}{3 \gamma^{2}-2 \gamma+1} \leq 1$ for $\gamma \in\left[\frac{1}{2}, 1\right]$.

The analysis so far holds only for $\gamma<1$ since for $\gamma=1$, division by zero occurs at some points in the analysis. For $\gamma=1$, we obtain a ratio of $2+\varepsilon=\frac{2 \gamma^{3}+2 \gamma^{2}}{3 \gamma^{2}-2 \gamma+1}+\varepsilon$ : We have $w(M) \leq\left(1+\frac{\varepsilon}{2}\right) \cdot w\left(S^{\prime}\right)$ and $w(T) \leq\left(1+\frac{\varepsilon}{2}\right) \cdot w\left(S^{\prime}\right)$, which implies the bound.

What remains to be proved is that the algorithm succeeds with a probability of at least $1 / 2$. First, we observe that if we iterate the randomized computation of an approximate Pareto curve, then we do not have to decide which set is indeed such an approximate Pareto curve. Instead, we can take the union of all solutions computed and remove all dominated solutions of the set thus obtained. The only randomization in Algorithm 2 is in the computation of the approximate Pareto curves for the matching problems. The number of curves to be computed is $p$, which is bounded by a polynomial of the input size and $1 / \varepsilon$. We can achieve a failure probability of at most $\frac{1}{2 p}$ by performing $\log (2 p)$ iterations of the FPRAS for the matching problem. The probability that one of the Pareto curve computations fails is thus at most $p \cdot \frac{1}{2 p}=1 / 2$, which completes the proof of the theorem.

We compare the ratios obtained by the two algorithms of this sections and the cycle cover algorithm of Section 4 in Section 5.1 . 


\section{Matchings and Cycle Covers}

A cycle cover of a graph is a spanning subgraph that consists solely of cycles such that every vertex is part of exactly one cycle. Many approximation algorithms for the singlecriterion TSP are based on cycle covers. These approximation algorithms usually start by computing an initial cycle cover and then join the cycles to obtain a Hamiltonian cycle. This technique is called subtour patching [15]. We show that there exist FPRASs for multi-criteria cycle cover problems.

ACC, the cycle cover problem in directed graphs, is equivalent to finding matchings of minimum weight in bipartite graphs (assignment problem). An FPRAS for the multicriteria matching problem is also an FPRAS for the multi-criteria matching problem in bipartite graphs. Hence, multi-criteria ACC also admits an FPRAS.

Theorem 3.1. There exists an FPRAS for multi-criteria ACC.

To show that multi-criteria SCC admits an FPRAS, we show that arbitrary graph factor problems admit FPRASs. Let $G=(V, E)$ be a graph and $f: V \rightarrow \mathbb{N}$ be function. A subset $F \subseteq E$ is called an $\boldsymbol{f}$-factor of $G$ if all vertices $v \in V$ have a degree of exactly $f(v)$ in the graph $(V, F)$.

Cycle covers of undirected graphs are also known as two-factors since every vertex is incident to exactly two edges. Thus, they are a special case of graph factors.

The graph factor problem GFP is the following minimization problem: An instance is an undirected graph $G=(V, E)$ with a function $f: V \rightarrow \mathbb{N}$ and an edge weight function $w: E \rightarrow \mathbb{N}$. The aim is to find an $f$-factor of minimum weight.

To show that multi-criteria GFP, and thus multi-criteria SCC as well, admits an FPRAS, we exploit Tutte's reduction [25], which reduces arbitrary graph factor problems to matchings (matchings are also known as one-factors since every vertex is incident to exactly one edge of the matching). We omit a description of the reduction, but refer to Lovász and Plummer [19] or Tutte [25] for the details. Overall, we obtain the following result.

Theorem 3.2. Multi-criteria GFP and multi-criteria SCC admit an FPRAS.

\section{Approximations Based on Cycle Covers}

\subsection{The Algorithm}

The generic outline of a cycle-cover-based algorithm is the following: Start by computing a cycle cover. Then remove one edge of every cycle. Finally, join the paths thus obtained to form a Hamiltonian cycle.

Algorithm 3 is our generalization of this algorithm to multi-criteria TSP. It achieves a constant approximation ratio if the quotient of the weight of the heaviest edge and the weight of the lightest edge is bounded.

In this section, we present a general analysis of the approximation ratio of this algorithm. We will refine the analysis for multi-criteria $\Delta(\gamma)$-STSP (Section 4.2) to get an improved approximation ratio. Furthermore, we apply the analysis to get approximation results for multi-criteria $\Delta(\gamma)$-ATSP (Section 4.3) and $\operatorname{STSP}(1,2)$ and $\operatorname{ATSP}(1,2)$ (Section 4.4). We analyze Algorithm 3 in terms of the number $\alpha n$ of edges that have to be removed and the quotient $\beta=w_{\max } / w_{\min }$.

Lemma 4.1. Assume that at most $\alpha$ n edges have to be removed from each cycle cover and that $\frac{\max _{e \in E} w_{i}(e)}{\min _{e \in E} w_{i}(e)} \leq \beta$ for all $i \in[k]$. 


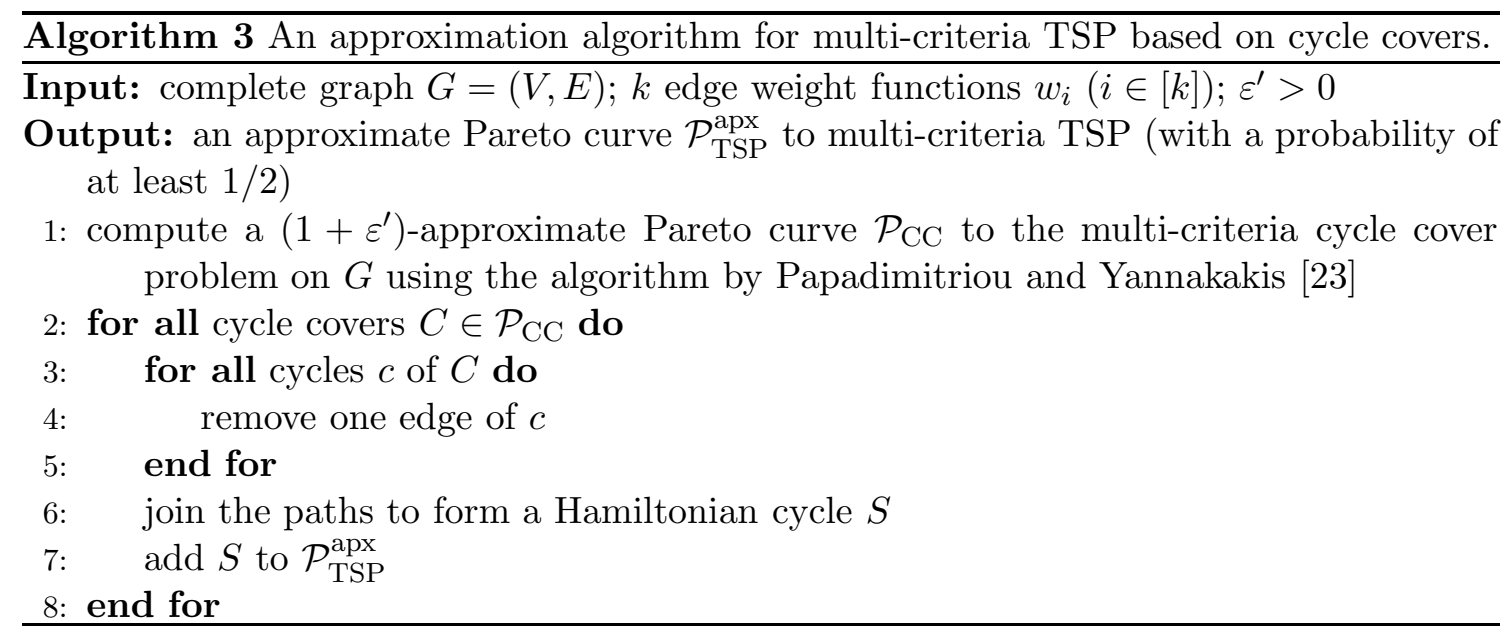

Then Algorithm 3 is a randomized $(1+\alpha(\beta-1)+\varepsilon)$ approximation algorithm for every $\varepsilon>0$. Its running-time is polynomial in the input size and $1 / \varepsilon$.

Proof. Without loss of generality, let $\min _{e \in E} w_{i}(e)=1$ for all $i \in[k]$. We run the algorithm with some $\varepsilon^{\prime}$ that depends on $\alpha, \beta$, and $\varepsilon$ and that we will specify later on. Let $S^{\prime}$ be an arbitrary Hamiltonian cycle. Then there exists a cycle cover $C$ in $\mathcal{P}_{\mathrm{CC}}$ with $w(C) \leq$ $\left(1+\varepsilon^{\prime}\right) \cdot w\left(S^{\prime}\right)$. We obtain a Hamiltonian cycle $S$ from $C$ such that

$$
w_{i}(S) \leq w_{i}(C)+\alpha n(\beta-1)
$$

for all $i \in[k]$. The reason for this is that every edge removed has a weight of at least 1 and every edge added has a weight of at most $\beta$. Now we have for all $i \in[k]$

$$
\begin{aligned}
\frac{w_{i}(S)}{w_{i}\left(S^{\prime}\right)} & \leq\left(1+\varepsilon^{\prime}\right) \cdot \frac{w_{i}(S)}{w_{i}(C)} \leq\left(1+\varepsilon^{\prime}\right) \cdot \frac{w_{i}(C)+\alpha n(\beta-1)}{w_{i}(C)} \\
& \leq\left(1+\varepsilon^{\prime}\right) \cdot \frac{n+\alpha n(\beta-1)}{n}=\left(1+\varepsilon^{\prime}\right) \cdot(1+\alpha(\beta-1)) \\
& \leq 1+\alpha(\beta-1)+\varepsilon
\end{aligned}
$$

for $\varepsilon^{\prime} \leq \frac{\varepsilon}{1+\alpha(\beta-1)}$, which proves the lemma.

\subsection{Refined Analysis for $\Delta(\gamma)$-STSP}

From the general analysis (Lemma 4.1), we obtain an approximation ratio of $\frac{2}{3}+\frac{2}{3}$. $\frac{\gamma^{2}}{1-\gamma}+\varepsilon$ for $\Delta(\gamma)$-STSP. In this section, we present a refined analysis that yields a better approximation ratio.

Consider any cycle $c$ of a cycle cover of $\mathcal{P}_{\mathrm{CC}}$. Then there will be an edge $e_{R}$ of $c$ that will be removed and an edge $e_{A}$ adjacent to $e_{R}$ that will be added during the joining process. Finally, there exists an edge $e_{K}$ of $c$ that is adjacent to both $e_{R}$ and $e_{A}$ (Figure 1 shows an example). Note that while $e_{R}$ is uniquely determined, once the edges have been removed and the new edges have been added, the edge $e_{A}$ is not since there are two edges that connect $c$ to other cycles of the cycle cover. However, once we have fixed $e_{A}$ for one cycle $c$, the corresponding $e_{K}$ is uniquely determined, and the $e_{A}^{\prime}$ and $e_{K}^{\prime}$ of all other cycles $c^{\prime}$ are also determined.

By Lemma 2.1, we have $w_{i}\left(e_{R}\right) \geq \frac{1-\gamma}{\gamma} \cdot w_{i}\left(e_{A}\right)$ and $w_{i}\left(e_{K}\right) \geq \frac{1-\gamma}{\gamma} \cdot w_{i}\left(e_{A}\right)$. All arguments in the following hold for all weight functions simultaneously. Thus, we restrict ourselves to considering one fixed weight function $w_{i}$ for some $i \in[k]$ to simplify the arguments. 


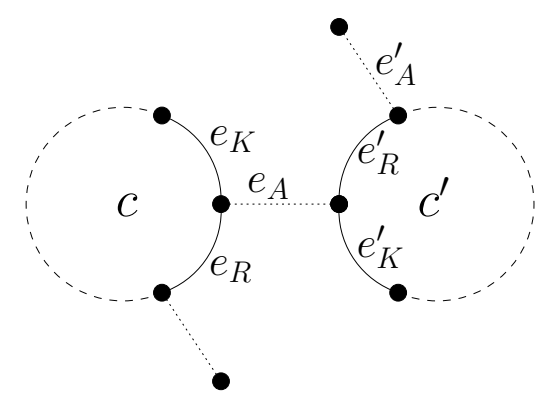

(a) Cycle cover, before the patching.

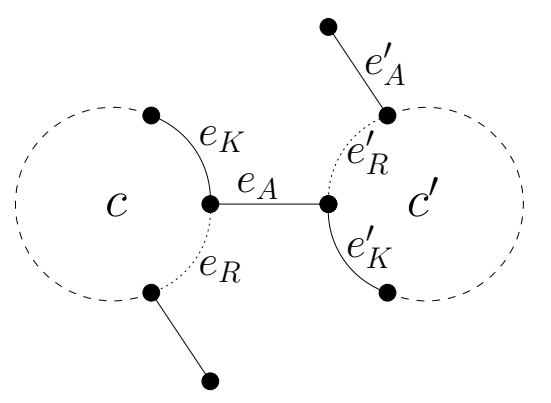

(b) Hamiltonian cycle, after the patching.

Figure 1: Two cycles $c$ and $c^{\prime}$ before and after joining the cycles to a Hamiltonian cycle. The edges $e_{R}, e_{K}$, and $e_{A}$ belong to $c$ while $e_{R}^{\prime}, e_{K}^{\prime}$, and $e_{A}^{\prime}$ belong to $c^{\prime}$.

Let $w_{R}$ be the total weight of edges removed, $w_{A}$ be the total weight of edges added, and $w_{K}$ be the total weight of edges of $C$ and $S$ that are adjacent to edges added. Then we have $w(C)=w+w_{K}+w_{R}$ for some suitably chosen $w \geq 0$, which is the total weight of all edges not taken into account so far. Thus,

$$
\frac{w_{i}(S)}{w_{i}(C)}=\frac{w+w_{K}+w_{A}}{w+w_{K}+w_{R}}=1+\frac{w_{A}-w_{R}}{w+w_{K}+w_{R}}=R
$$

Since $R$ is monotonically decreasing with respect to $w_{R}$, we obtain

$$
R \leq 1+\frac{w_{A}-\frac{1-\gamma}{\gamma} \cdot w_{A}}{w+w_{K}+\frac{1-\gamma}{\gamma} \cdot w_{A}}=R^{\prime} .
$$

Exploiting further that $R^{\prime}$ is monotonically decreasing in $w_{K}$, we get

$$
R^{\prime} \leq 1+\frac{w_{A}-\frac{1-\gamma}{\gamma} \cdot w_{A}}{w+\frac{1-\gamma}{\gamma} \cdot 2 w_{A}}=1+\frac{w_{A}(2 \gamma-1)}{\gamma w+(1-\gamma) 2 w_{A}}=R^{\prime \prime} .
$$

The inequalities $w_{A} \leq \frac{2 \gamma^{2} n}{3(1-\gamma)}$ and $w \geq n / 3$ hold since every cycle has a length of at least three. We exploit the fact that $R^{\prime \prime}$ is monotonically increasing with respect to $w_{A}$ and monotonically decreasing with respect to $w$ :

$$
R^{\prime \prime} \leq 1+\frac{\frac{2 \gamma^{2} n}{3(1-\gamma)} \cdot(2 \gamma-1)}{\frac{\gamma n}{3}+(1-\gamma) \cdot 2 \cdot \frac{2 \gamma^{2} n}{3(1-\gamma)}}=1+\frac{\frac{2 \gamma^{2}}{1-\gamma} \cdot(2 \gamma-1)}{\gamma+4 \gamma^{2}}=\frac{1+\gamma}{1+3 \gamma-4 \gamma^{2}}
$$

We run the algorithm with some $\varepsilon^{\prime}>0$ that depends on $\gamma$. We will specify $\varepsilon^{\prime}$ in a moment. Let $S^{\prime}$ be an arbitrary Hamiltonian cycle and $C \in \mathcal{P}_{\mathrm{CC}}$ be a cycle cover with $w_{i}(C) \leq\left(1+\varepsilon^{\prime}\right) \cdot w\left(S^{\prime}\right)$ for all $i \in[k]$. Let $S$ be the Hamiltonian cycle obtained from $C$. Then

$$
w_{i}(S) \leq\left(1+\varepsilon^{\prime}\right) \cdot \frac{1+\gamma}{1+3 \gamma-4 \gamma^{2}} \cdot w_{i}\left(S^{\prime}\right)
$$

For a given $\varepsilon>0$, we choose $\varepsilon^{\prime}$ such that $\varepsilon^{\prime} \cdot \frac{1+\gamma}{1+3 \gamma-4 \gamma^{2}} \leq \varepsilon$. The set $\mathcal{P}_{\text {TSP }}^{\text {apx }}$ is a $\left(\frac{1+\gamma}{1+3 \gamma-4 \gamma^{2}}+\varepsilon\right)$ approximate Pareto curve with a probability of at least $1 / 2$, which implies the following theorem.

Theorem 4.2. For $\gamma \in\left[\frac{1}{2}, 1\right)$, Algorithm 3 is a randomized $\left(\frac{1+\gamma}{1+3 \gamma-4 \gamma^{2}}+\varepsilon\right)$-approximation algorithm for all $\varepsilon>0$. Its running-time is polynomial in the input size and $1 / \varepsilon$.

In Section 5.1, we compare the approximation ratios of the cycle cover algorithm for $\Delta(\gamma)$-STSP to the tree doubling and Christofides' algorithm. 


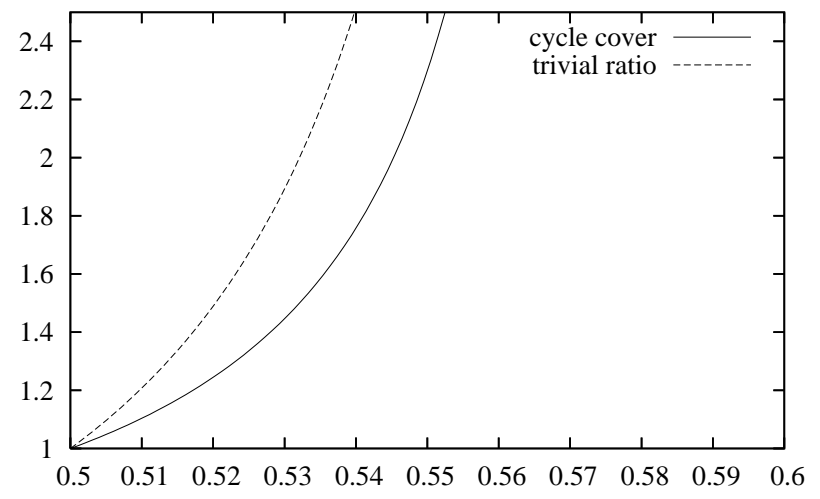

Figure 2: The approximation ratio of Algorithm 3 achieved for $\Delta(\gamma)$-ATSP subject to $\gamma$ compared to the trivial approximation ratio of $w_{\max } / w_{\min }$.

\subsection{The Cycle Cover Algorithm for $\Delta(\gamma)$-ATSP}

For multi-criteria $\Delta(\gamma)$-ATSP, our algorithm yields a constant factor approximation if $\gamma<\frac{1}{\sqrt{3}}$ since $w_{\max } / w_{\min }$ is bounded from above by $\frac{2 \gamma^{3}}{1-3 \gamma^{2}}$ for such $\gamma$. For larger values of $\gamma$, this ratio can be unbounded.

Lemma 4.3 (Chandran and Ram [9]). Let $\gamma \in[1 / 2,1)$. Let $G=(V, E)$ be a directed complete graph, and let $w: E \rightarrow \mathbb{N}$ be an edge weight function satisfying $\gamma$-triangle inequality. Let $w_{\min }=\min _{e \in E} w(e)$ and $w_{\max }=\max _{e \in E} w(e)$.

If $\gamma<1 / \sqrt{3}$, then $\frac{w_{\max }}{w_{\min }} \leq \frac{2 \gamma^{3}}{1-3 \gamma^{2}}$. If $\gamma \geq 1 / \sqrt{3}$, then $\frac{w_{\max }}{w_{\min }}$ can be unbounded.

By combining Lemma 4.1 and Lemma 4.3, we obtain the following result.

Theorem 4.4. For $\gamma<1 / \sqrt{3}$, Algorithm 3 is a randomized $\left(\frac{1}{2}+\frac{\gamma^{3}}{1-3 \gamma^{2}}+\varepsilon\right)$-approximation algorithm for $\Delta(\gamma)$-ATSP. Its running-time is polynomial in the input size and $1 / \varepsilon$.

Figure 2 shows the approximation ratio achieved for multi-criteria $\Delta(\gamma)$-ATSP subject to $\gamma$ and compared to the trivial ratio of $w_{\max } / w_{\max }$.

We leave as an open problem to generalize the analysis to larger values of $\gamma$. However, it seems to be hard to find a constant factor approximation for $\gamma=1$, i. e., for multicriteria $\Delta$-ATSP, since this would immediately yield a constant factor approximation for single-criterion $\Delta$-ATSP.

\subsection{TSP with Weights One and Two}

Now we analyze the cycle cover algorithm for multi-criteria TSP with weights one and two. For both $\operatorname{STSP}(1,2)$ and $\operatorname{ATSP}(1,2)$, we have $\beta=2$, i. e., $w_{\max } / w_{\min }=2$. Furthermore, for $\operatorname{STSP}(1,2)$, we have $\alpha \leq 1 / 3$, while we only have $\alpha \leq 1 / 2$ in case of $\operatorname{ATSP}(1,2)$. The approximation ratio follows by exploiting Lemma 4.1 .

Note that the edge weights and thus the objective functions are polynomially bounded for $\operatorname{STSP}(1,2)$ and $\operatorname{ATSP}(1,2)$. Thus, we can compute a Pareto curve of cycle covers instead of only a $(1+\varepsilon)$-approximate Pareto curve. This implies that we do not have an additional $\varepsilon$ in the approximation ratios in the following theorems.

Theorem 4.5. Algorithm 3 is a randomized 4/3-approximation algorithm for multi-criteria $\operatorname{STSP}(1,2)$. Its running-time is polynomial. 


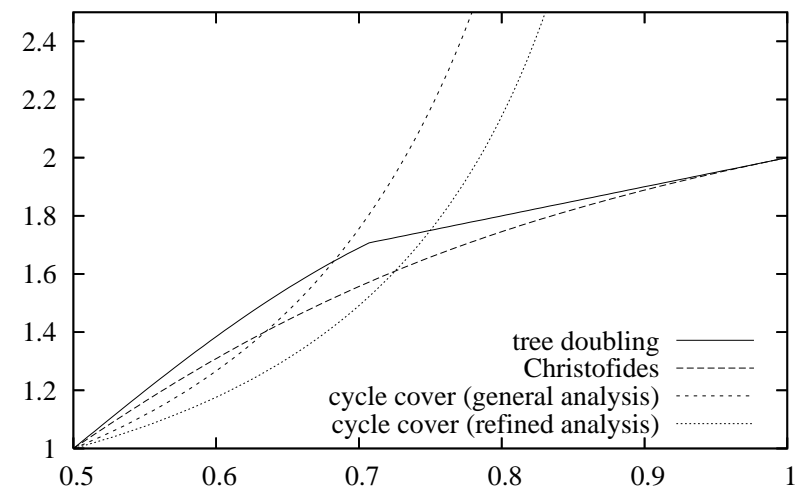

Figure 3: Approximation ratios subject to $\gamma$ achieved by the tree doubling algorithm (Algorithm 1), Christofides' algorithm (Algorithm 2), and the cycle cover algorithm (Algorithm 3, Section 4), for which both the ratio obtained from the general analysis (Section 4.1) and from the refined analysis (Section 4.2) are shown.

Theorem 4.6. Algorithm 3 is a randomized 3/2-approximation algorithm for multi-criteria $\operatorname{ATSP}(1,2)$. Its running-time is polynomial.

\section{Concluding Remarks}

\subsection{Comparing the Approximation Ratios}

Let us compare the approximation ratios for $\Delta(\gamma)$-STSP achieved by the tree doubling algorithm (Algorithm 1), Christofides' algorithm (Algorithm 2), and the cycle cover algorithm (Algorithm 3).

Figure 3 shows the approximation ratios achieved by these algorithms subject to $\gamma$. Figure 4 shows the approximation ratios achieved deterministically (by the tree doubling algorithm) and randomized (by a combination of Christofides' and the cycle cover algorithm). The ratios are compared to the trivial ratio of $w_{\max } / w_{\min }$ and to the currently best known approximation ratio for single-criterion $\Delta(\gamma)$-STSP. Note that in particular for small values of $\gamma$, our algorithms for multi-criteria $\Delta(\gamma)$-STSP come close to achieving the ratio of the best algorithms for single-criterion $\Delta(\gamma)$-STSP.

\subsection{Open Problems}

Our approximation algorithm for multi-criteria $\Delta(\gamma)$-ATSP works only for $\gamma<1 / \sqrt{3}$. Thus, we are interested in finding constant factor approximation algorithms also for $\gamma \geq$ $1 / \sqrt{3}$, which exist for all $\gamma<1$ for single-criterion $\Delta(\gamma)$-ATSP $[7,9]$

The cycle-cover-based algorithm for Max-TSP, where Hamiltonian cycles of maximum weight are sought, does not seem to perform well for multi-criteria Max-TSP. The reason for this is that the approximation algorithms for Max-TSP that base on cycle covers usually contain a statement like "remove the lightest edge of every cycle". While this works for single-criterion TSP, the term "lightest edge" is not well-defined for multi-criteria traveling salesman problems. We are particularly curious about the approximability of multi-criteria Max-TSP. 


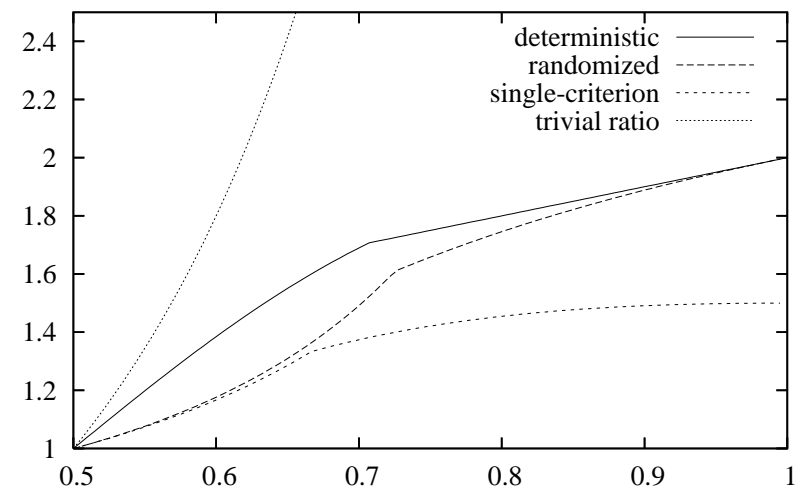

Figure 4: Approximation ratios subject to $\gamma$. The deterministic ratio is achieved by the tree doubling algorithm. Combining Christofides' and the cycle cover algorithm yields the randomized ratio. For comparison, the current ratio for single-criterion $\Delta(\gamma)$-STSP and the trivial ratio $w_{\max } / w_{\min }$ are also shown.

\section{Acknowledgments}

We thank Jan Arpe for valuable comments.

\section{References}

[1] Eric Angel, Evripidis Bampis, and Laurent Gourvés. Approximating the Pareto curve with local search for the bicriteria TSP $(1,2)$ problem. Theoretical Computer Science, 310(1-3):135-146, 2004.

[2] Eric Angel, Evripidis Bampis, Laurent Gourvès, and Jérøme Monnot. (Non-)approximability for the multi-criteria TSP $(1,2)$. In Maciej Liśkiewicz and Rüdiger Reischuk, editors, Proc. of the 15th Int. Symp. on Fundamentals of Computation Theory (FCT), volume 3623 of Lecture Notes in Computer Science, pages 329-340. Springer, 2005.

[3] Giorgio Ausiello, Pierluigi Crescenzi, Giorgio Gambosi, Viggo Kann, Alberto Marchetti-Spaccamela, and Marco Protasi. Complexity and Approximation: Combinatorial Optimization Problems and Their Approximability Properties. Springer, 1999.

[4] Francisco Barahona and William R. Pulleyblank. Exact arborescences, matchings and cycles. Discrete Applied Mathematics, 16(2):91-99, 1987.

[5] Piotr Berman and Marek Karpinski. 8/7-approximation algorithm for (1,2)-TSP. In Proc. of the 17th Ann. ACM-SIAM Symp. on Discrete Algorithms (SODA), pages 641-648. SIAM, 2006.

[6] Markus Bläser. A 3/4-approximation algorithm for maximum ATSP with weights zero and one. In Klaus Jansen, Sanjeev Khanna, José D. P. Rolim, and Dana Ron, editors, Proc. of the 7th Int. Workshop on Approximation Algorithms for Combinatorial Optimization Problems (APPROX), volume 3122 of Lecture Notes in Computer Science, pages 61-71. Springer, 2004.

[7] Markus Bläser, Bodo Manthey, and Jiř́ Sgall. An improved approximation algorithm for the asymmetric TSP with strengthened triangle inequality. Journal of Discrete Algorithms, 4(4):623-632, 2006. 
[8] Hans-Joachim Böckenhauer, Juraj Hromkovič, Ralf Klasing, Sebastian Seibert, and Walter Unger. Approximation algorithms for the TSP with sharpened triangle inequality. Information Processing Letters, 75(3):133-138, 2000.

[9] L. Sunil Chandran and L. Shankar Ram. On the relationship between ATSP and the cycle cover problem. Theoretical Computer Science, 370(1-3):218-228, 2007.

[10] Nicos Christofides. Worst-case analysis of a new heuristic for the traveling salesman problem. Technical Report 388, Graduate School of Industrial Administration, Carnegie Mellon University, Pittsburgh, Pennsylvania, USA, 1976.

[11] Matthias Ehrgott. Approximation algorithms for combinatorial multicriteria optimization problems. International Transactions in Operational Research, 7(1):5-31, 2000 .

[12] Matthias Ehrgott. Multicriteria Optimization. Springer, 2005.

[13] Matthias Ehrgott and Xavier Gandibleux. A survey and annotated bibliography of multiobjective combinatorial optimization. OR Spectrum, 22(4):425-460, 2000.

[14] Michael R. Garey and David S. Johnson. Computers and Intractability: A Guide to the Theory of NP-Completeness. W. H. Freeman and Company, 1979.

[15] Paul C. Gilmore, Eugene L. Lawler, and David B. Shmoys. Well-solved special cases. In Lawler et al. [18], pages 87-143.

[16] Gregory Gutin and Abraham P. Punnen, editors. The Traveling Salesman Problem and its Variations. Kluwer Academic Publishers, 2002.

[17] Haim Kaplan, Moshe Lewenstein, Nira Shafrir, and Maxim I. Sviridenko. Approximation algorithms for asymmetric TSP by decomposing directed regular multigraphs. Journal of the ACM, 52(4):602-626, 2005.

[18] Eugene L. Lawler, Jan Karel Lenstra, Alexander H. G. Rinnooy Kan, and David B. Shmoys, editors. The Traveling Salesman Problem: A Guided Tour of Combinatorial Optimization. John Wiley \& Sons, 1985.

[19] László Lovász and Michael D. Plummer. Matching Theory, volume 121 of NorthHolland Mathematics Studies. Elsevier, 1986.

[20] Bodo Manthey and L. Shankar Ram. Approximation algorithms for multi-criteria traveling salesman problems. In Thomas Erlebach and Christos Kaklamanis, editors, Proc. of the 4th Workshop on Approximation and Online Algorithms (WAOA 2006), volume 4368 of Lecture Notes in Computer Science, pages 302-315. Springer, 2007.

[21] Ketan Mulmuley, Umesh V. Vazirani, and Vijay V. Vazirani. Matching is as easy as matrix inversion. Combinatorica, 7(1):105-113, 1987.

[22] Christos H. Papadimitriou. The complexity of restricted spanning tree problems. Journal of the ACM, 29(2):285-309, 1982.

[23] Christos H. Papadimitriou and Mihalis Yannakakis. On the approximability of tradeoffs and optimal access of web sources. In Proc. of the 41st Ann. IEEE Symp. on Foundations of Computer Science (FOCS), pages 86-92. IEEE Computer Society, 2000. 
[24] Daniel J. Rosenkrantz, Richard E. Stearns, and Philip M. Lewis II. An analysis of several heuristics for the traveling salesman problem. SIAM Journal on Computing, 6(3):563-581, 1977.

[25] William T. Tutte. A short proof of the factor theorem for finite graphs. Canadian Journal of Mathematics, 6:347-352, 1954.

[26] Vijay V. Vazirani. Approximation Algorithms. Springer, 2001. 